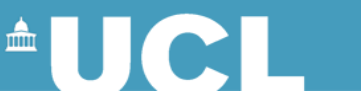

\section{Minor roads can also be difficult to cross Can we rely on driver courtesy?}

\author{
Paulo Rui Anciaes ${ }^{1}$ \\ Giovanni Di Guardo² \\ Peter Jones $^{1}$

\footnotetext{
${ }^{1}$ Centre for Transport Studies, UCL (University College London)

${ }^{2}$ Transport Planning Associates, UK
}

$6^{\text {th }}$ International Conference on Transport and Health 18-06-2021

\section{Roads are barriers to health}




\section{Minor roads are barriers too}
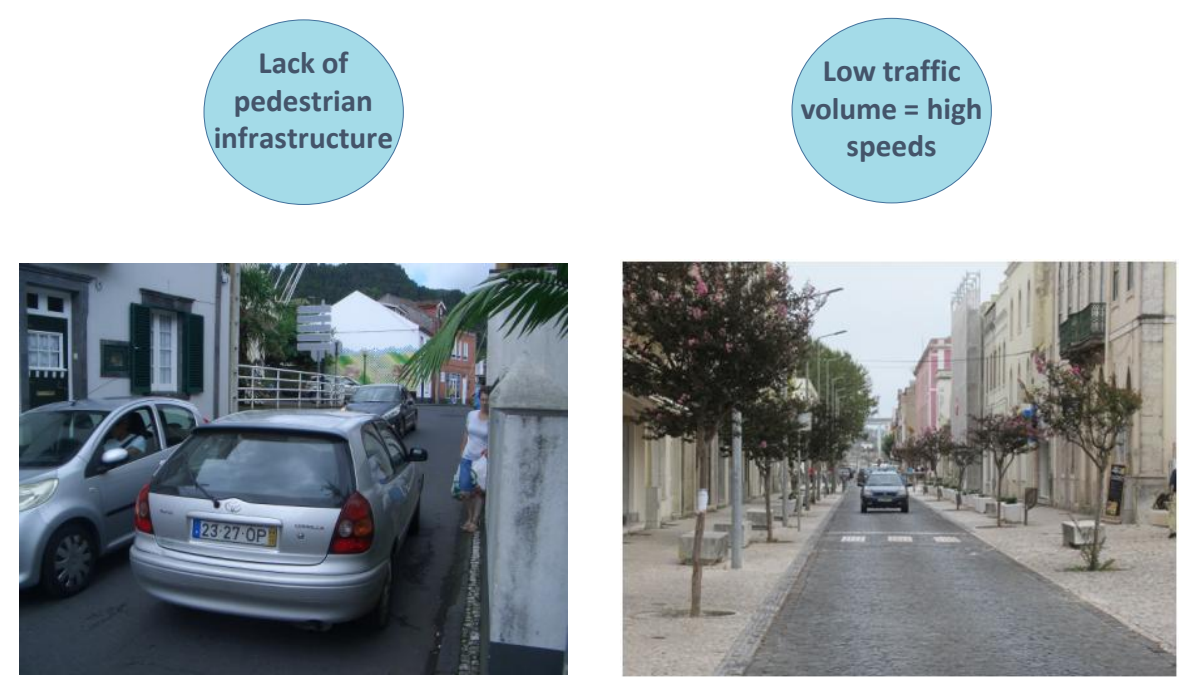

A common solution on minor roads: zebras (marked crosswalks)
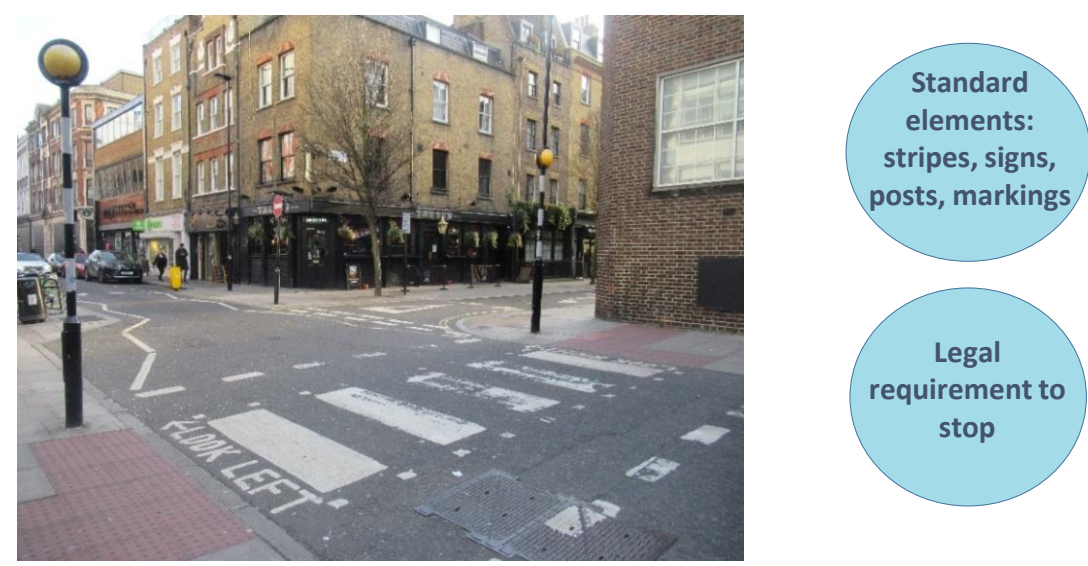

Poor safety record

(lack of driver compliance? pedestrians unwarranted sense of safety?) 


\section{Another solution: courtesy crossings}

Drivers not legally required to stop for pedestrians but encouraged to do so by design elements

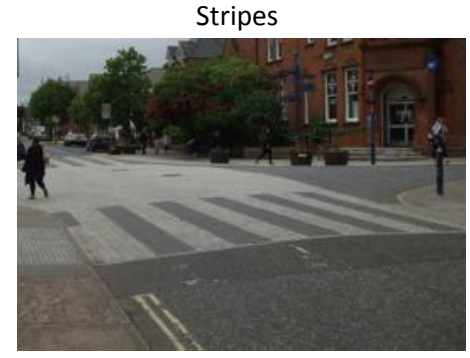

Visual narrowing

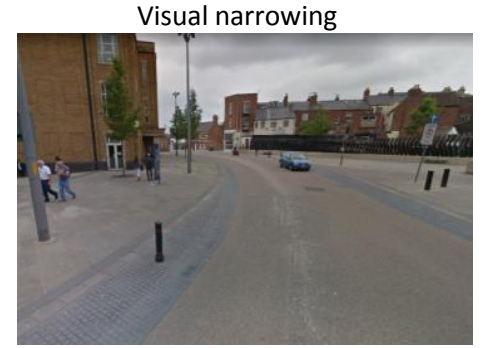

Source of stripes and visual narrowing photos: $\mathrm{ClHT}$ (2018)
Colours/textures

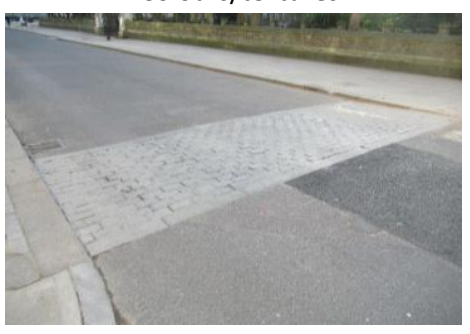

Ramps

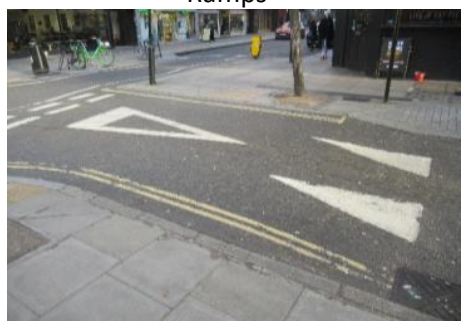

\section{Courtesy crossings are controversial}

Some reports that drivers and pedestrians feel confused

Little evidence on how these crossings address movement and safety of pedestrians

No guidance on where to provide and how to design these crossings.

Guidance from UK Dept. Transport withdrawn in 2018, seeking more research

\section{Questions answered in this presentation}

What are the factors that encourage drivers to stop for pedestrians at courtesy crossings?

Which design elements are more effective? 


\section{Data}

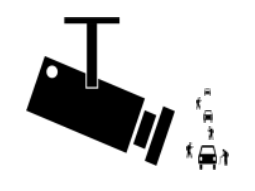

937 interactions between drivers and pedestrians at 23 crossings in England (with various combinations of courtesy design elements)

One possible interaction for each separate traffic lane pedestrians cross

\section{Variables}

\section{Design elements}

\section{Stripes}

Colours/textures

Visual narrowing

Ramps

Crossing stage

From median strip to footway, From footway to median strip, or From

footway to footway

First Lane, Second Lane, or Second

Lane (opposite direction)

\section{Pedestrian situation}

Single pedestrian/group

No others, Others crossing ahead, or

Others crossing from opposite side

Pedestrian characteristics

\section{Gender}

Age (child, younger adult, older adult)

Mobility restrictions

Vehicle situation/characteristics

Followed by another vehicle

Large vehicle (HGV/bus)

\section{Infrastructure characteristics}

Link, Junction (inbound), or Junction (outbound)

Speed limit

Raised kerb or not

Site characteristics

Shops or not

Time/day

Peak or not peak

Weekday or Saturday

\section{Average courtesy rates, by type of crossing}

\begin{tabular}{|c|c|c|c|c|c|c|c|}
\hline \multirow[b]{2}{*}{ Zebra } & \multicolumn{4}{|c|}{ Courtesy crossing design elements } & \multirow[b]{2}{*}{$\begin{array}{l}\text { Number } \\
\text { of crossings }\end{array}$} & \multirow{2}{*}{$\begin{array}{c}\% \text { interactions } \\
\text { where first } \\
\text { vehicle stops }\end{array}$} & \multirow{2}{*}{$\begin{array}{c}\% \text { interactions } \\
\text { where any } \\
\text { vehicle stops }\end{array}$} \\
\hline & $\begin{array}{c}\text { Stripes } \\
\text { (not zebra) }\end{array}$ & $\begin{array}{l}\text { Colours/ } \\
\text { textures }\end{array}$ & $\begin{array}{c}\text { Visual } \\
\text { narrowing }\end{array}$ & Ramps & & & \\
\hline \multirow{10}{*}{$\mathrm{x}$} & & $x$ & & & 2 & 4 & 4 \\
\hline & & & $\mathrm{x}$ & & 1 & 20 & 42 \\
\hline & & $\mathrm{x}$ & $\mathrm{x}$ & & 4 & 54 & 54 \\
\hline & & $\mathrm{x}$ & $\mathrm{x}$ & $\mathrm{x}$ & 3 & 67 & 78 \\
\hline & & & & $x$ & 1 & 76 & 92 \\
\hline & & $\mathrm{x}$ & & $x$ & 3 & 76 & 84 \\
\hline & $\mathrm{x}$ & $\mathrm{x}$ & & $x$ & 4 & 78 & 88 \\
\hline & & & & & 3 & 88 & 96 \\
\hline & $\mathrm{x}$ & $\mathrm{x}$ & & & 1 & 95 & 95 \\
\hline & $\mathrm{x}$ & & $\mathrm{x}$ & & 1 & 97 & 99 \\
\hline & 23 & 73 & 81 \\
\hline
\end{tabular}




\section{Courtesy rates, by other characteristics}

\begin{tabular}{|c|c|c|c|}
\hline & & $\begin{array}{c}\text { First vehicle } \\
\text { stops (\%) }\end{array}$ & $\begin{array}{c}\text { Any vehicle } \\
\text { stops (\%) }\end{array}$ \\
\hline & & $\%$ & $\%$ \\
\hline \multirow[t]{3}{*}{ Crossing stage } & From median strip to footway & 80 & 90 \\
\hline & From footway to median strip & 79 & 89 \\
\hline & From footway to footway & 66 & 74 \\
\hline \multirow[t]{3}{*}{ Pedestrian situation } & No others & 64 & 74 \\
\hline & Others crossing ahead & 84 & 87 \\
\hline & Others crossing from opposite side & 85 & 88 \\
\hline \multirow[t]{2}{*}{ Vehicle situation } & Followed by another vehicle & 79 & 88 \\
\hline & Not followed & 59 & 66 \\
\hline \multirow[t]{2}{*}{ Vehicle characteristics } & Small vehicle (Car/motorcycle) & 70 & 78 \\
\hline & Large vehicle (HGV/bus) & 42 & 50 \\
\hline \multirow[t]{2}{*}{ Site characteristics } & Shops along footway & 75 & 83 \\
\hline & No shops & 64 & 72 \\
\hline \multirow[t]{3}{*}{ Day } & Weekday & 67 & 74 \\
\hline & Saturday & 77 & 87 \\
\hline & All & 69 & 77 \\
\hline
\end{tabular}

Insignificant differences: first lane vs. second lane, single pedestrian vs. group, gender, age, mobility restrictions, link vs. junction, speed limit, raised kerb

\section{Model results}

\begin{tabular}{llcc}
\hline & & \multicolumn{2}{c}{ First vehicle Any vehicle } \\
& Stops & stops \\
\hline Design elements & Constant & -4.31 & -9.04 \\
& Stripes (not zebra-like) & 1.68 & 1.80 \\
& Colours/textures & 1.35 & 1.11 \\
& Visual narrowing of carriageway & 1.38 & 3.00 \\
& Ramps & 1.47 & 2.21 \\
\hline Crossing stage & From median strip to footway & 2.29 & 5.14 \\
& From footway to median strip & 1.36 & 4.60 \\
& Second lane & 0.67 & \\
\hline Pedestrian situation & Group & 0.58 & \\
& Others crossing ahead & 1.39 & 0.63 \\
& Others crossing from other side & 1.96 & 1.39 \\
\hline Pedestrian characteristics & At least one woman & & 0.83 \\
\hline Vehicle situation & Followed by another vehicle & 0.69 & 1.41 \\
\hline Other infrastructure & Junction, inbound traffic & -1.29 & \\
Characteristics & Junction, outbound traffic & -1.37 & \\
& Speed limit=20mph & 1.14 & 4.31 \\
\hline Site characteristics & Shops & 0.90 & 2.40 \\
\hline
\end{tabular}

Insignificant variables: age, mobility restrictions, large vehicle, raised kerb, time, day 


\section{Before-after analysis}

Before

(visual narrowing of carriageway only)

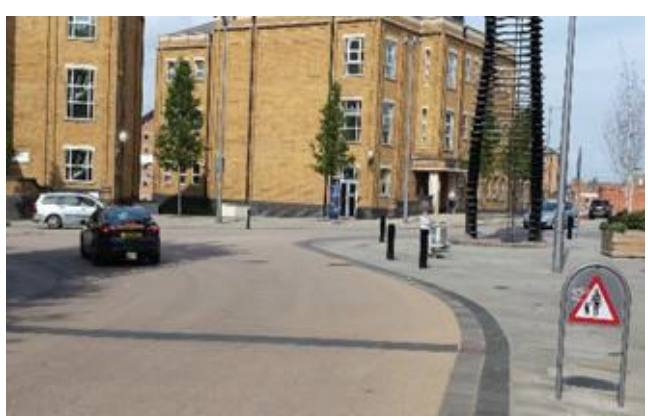

After

(visual narrowing of carriageway $\underline{\text { AND }}$ stripes)

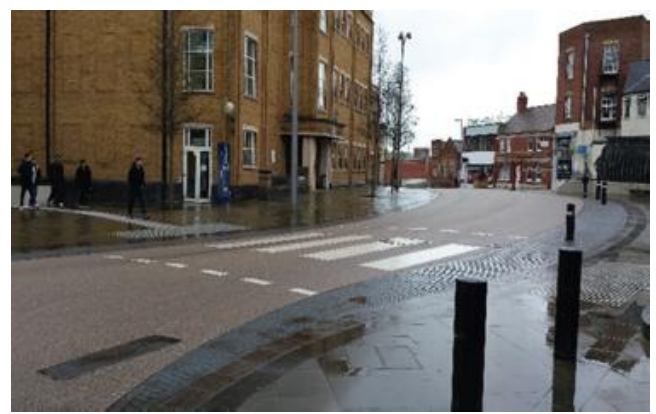

\section{Courtesy rates: before and after}

\begin{tabular}{lcccc}
\hline Variable & \multicolumn{3}{c}{ \% first vehicle stops } & \% any vehicle stops \\
\cline { 2 - 5 } & Before & After & Before & After \\
\hline Crossing stage & 16 & 96 & 39 & 99 \\
First Lane & 24 & 98 & 44 & 100 \\
Second Lane (in opposite direction) & & & & \\
\hline Pedestrian situation & 22 & 99 & 38 & 100 \\
Single pedestrian & 19 & 95 & 44 & 99 \\
Group & 16 & 94 & 38 & 99 \\
\hline No others & 33 & 100 & 67 & 100 \\
Others crossing ahead & 35 & 100 & 53 & 100 \\
Others crossing from oppposite side & & & & \\
Pedestrian characteristics & 22 & 95 & 48 & 99 \\
At least one woman & 17 & 99 & 28 & 100 \\
No women & 20 & 97 & 42 & 99 \\
\hline All & & &
\end{tabular}




\title{
Conclusions
}

All four design elements (stripes, colours/textures, visual narrowing, ramps) significantly increase courtesy rates

Some of the other factors increasing courtesy rates have also been found in previous literature to increase propensity to stop at zebras, e.g. crossing from/to median strip, lower speed limit

Weak/no evidence that courtesy behaviour is related to characteristics of pedestrians (age, gender, mobility restrictions)

\section{Implications}

Design of courtesy crossings should include all four design elements considered If possible, in combination with median strips and lower speed limits

\section{Thank you for your attention!}

\author{
Further information:
}

Anciaes, P., Di Guardo, G., Jones, P. (2020) Factors explaining driver yielding behaviour towards pedestrians at courtesy crossings.

Transportation Research F: Traffic Psychology and Behaviour 73, 453-469

https://iris.ucl.ac.uk/iris/browse/profile?upi=PRANC25 